\title{
Bone resorption levels are related to diet in UK dwelling South Asian but not Caucasian women
}

High levels of bone resorption are associated with increased risk of osteoporotic fractures ${ }^{(1)}$. The aim of the present study was to examine the predictive ability of diet and anthropometric factors in explaining bone resorption levels. This analysis used summer data for serum vitamin D status (25-hydroxyvitamin D [25(OH)D]), dietary nutrient intake, and anthropometry from the 2006-7 D-FINES Study (funded by the FSA, project N05064). This was a year-long study which investigated $n=375$ Surrey dwelling Caucasian and South Asian women (aged 18-65 years) in four seasons of the year. Dietary intake was assessed using $4 \mathrm{~d}$ estimated food diaries. Dietary protein to potassium ratio was included as an estimation of net acid excretion ${ }^{(2)}$. Bone resorption levels were measured by assessment of Cross-linked N-telopeptides of type I collagen [uNTX], from urine samples (funded by a grant from the National Osteoporosis Society (2011)). This was measured using the automated analyser Vitros ECi, Ortho Clinical Diagnostics (Rochester NY, USA) and adjusted for creatinine excretion. Multiple regression analysis examined the predictive ability of age, body mass index (BMI), log 25(OH)D and energy adjusted dietary calcium and vitamin D intakes in explaining uNTX (Table). 25(OH)D was log transformed to normalise distribution.

\begin{tabular}{|c|c|c|c|c|c|c|c|c|}
\hline \multirow[b]{2}{*}{ uNTX (BCE/mmol creatinine) } & \multicolumn{4}{|c|}{ SOUTH ASIAN $n=44$} & \multicolumn{4}{|c|}{ CAUCASIAN $n=226$} \\
\hline & $\mathrm{B}^{*}$ & $\mathrm{SE}$ & Beta** & $P$ & $\mathrm{~B}^{*}$ & $\mathrm{SE}$ & Beta** & $P$ \\
\hline Constant & $229 \cdot 2$ & $47 \cdot 8$ & - & $<0.001$ & $68 \cdot 6$ & $36 \cdot 0$ & - & $0 \cdot 06$ \\
\hline Age (years) & $0 \cdot 6$ & 0.4 & $0 \cdot 2$ & $0 \cdot 18$ & 0.8 & $0 \cdot 2$ & $0 \cdot 3$ & $<0 \cdot 001$ \\
\hline $\mathrm{BMI} \mathrm{kg} / \mathrm{m}^{2}$ & $-1 \cdot 9$ & 1.0 & $-0 \cdot 3$ & $0 \cdot 07$ & $-1 \cdot 0$ & $0 \cdot 5$ & $-0 \cdot 1$ & 0.04 \\
\hline $25(\mathrm{OH}) \mathrm{D} \mathrm{nmol} / \mathrm{L}$ & $-75 \cdot 3$ & $21 \cdot 3$ & $-0 \cdot 5$ & $<0.001$ & $-19 \cdot 2$ & $16 \cdot 1$ & $-0 \cdot 1$ & $0 \cdot 24$ \\
\hline Energy adjusted Diet $\mathrm{Ca} \mathrm{mg} / \mathrm{Kcal}$ & $-465 \cdot 7$ & $177 \cdot 2$ & $-0 \cdot 4$ & $0 \cdot 01$ & $51 \cdot 3$ & $74 \cdot 8$ & 0.04 & 0.49 \\
\hline Energy adjusted Diet Vit D mcg/Kcal & $-9954 \cdot 2$ & $20769 \cdot 1$ & $-0 \cdot 1$ & 0.64 & $12595 \cdot 8$ & 7233.9 & $0 \cdot 1$ & 0.08 \\
\hline Dietary Prot.:K ratio & $220 \cdot 7$ & $848 \cdot 4$ & 0.04 & $0 \cdot 80$ & $-24 \cdot 7$ & $374 \cdot 6$ & $0 \cdot 0$ & 0.95 \\
\hline ANOVA & $\mathrm{R}^{2}=0 \cdot 372$ & adj. $R^{2}=0.271$ & $p=0.006$ & & $\mathrm{R}^{2}=0 \cdot 139$ & $\operatorname{adj} R^{2}=0 \cdot 116$ & $p<0.001$ & \\
\hline
\end{tabular}

* = Unstandardised $; *=$ Standardised $;$ BMI = Body mass index $;$ adj.$=$ adjusted $;$ Prot.$=$ Protein $;$ K = Potassium

In South Asians, the model explained $27.1 \%$ of variance in UNTX, with the only statistically significant parameters being log 25 $(\mathrm{OH}) \mathrm{D}(p<0.001)$ and energy adjusted dietary calcium $(p=0.01)$. There was no significant predictive ability of age or BMI in explaining UNTX. In Caucasians, the model explained $11.6 \%$ of UNTX, with age $(p<0.001)$ and BMI $(p=0.04)$ being the only statistically significant predictors. Age and BMI not being predictors of bone resorption levels is the South Asian group is surprising, as postmenopausal women are known to have much higher levels of uNTX than premenopausal women. This finding is novel and warrants further investigation as if not offset by increased bone formation, this increased bone resorption may indicate a serious detriment to the bone health of premenopausal Asian women. This analysis suggests that dietary calcium and vitamin D status are potentially important factors for bone health in South Asian women particularly, who might benefit from public health measures to increase calcium intake and reduce vitamin D deficiency.

The D-FINES study was funded by the UK Food standards Agency (Project N05064). Measurement of uNTX (bone resorption marker) was funded by the National Osteoporosis Society. All views are those of the authors alone.

1. Cauley JA, Danielson ME, Greendale GA et al. (2012) Menopause 19, 1200-7.

2. Frassetto L, Todd K, Morris RC et al. (1998) Am J Clin Nutr 68, 576-583. 